Article

\title{
The Analysis and Calculation of DC Earth Electrode Electric Field Coupling with Thermal based on Shell Theory
}

\author{
Jingli Li ${ }^{1 *}$, Liying Guo ${ }^{2}$, Peng $\mathrm{Hu}^{2}$, Yuanbo $\mathrm{Li}^{3}$, Yu Zhang ${ }^{1}$ and Pengwei He ${ }^{1}$ \\ 1 College of Electrical Engineering, Zhengzhou University, Zhengzhou 450001, Henan Province, China; \\ lijingli1022@zzu.edu.cn \\ 2 Xinyang Electric Company, State Grid Henan Electric Power Company, Xinyang 464000, China; \\ guoliyingyuer@163.com \\ 3 Heze Electric Company, State Grid Shandong Electric Power Company, Heze 274000, China; \\ liyuanbo6154@163.com \\ * Correspondence: lijingli1022@zzu.edu.cn; Tel.: +86-158-901-60795
}

\begin{abstract}
During HVDC earth return operation systems, a high magnitude current will be injected into soil through earth electrode, the potential on the surface would change widely and produce unfavorable effects on the AC systems around. This paper presents an effective finite element method (FEM) coupling electric field with thermal field to evaluate the electrical field induced by the injected DC current. Firstly, owe to the characteristic of FEM, this method can consider arbitrary soil and earth electrode structure. Secondly, by setting the electrical and thermal parameters of soil as a function of temperature at the same time, the dynamic coupling process of electric field and thermal field is simulated accurately. Thirdly, to deal with the singular point in FEM subdivision and the huge computation in traditional three-dimensional FEM, the FEM coupling 2-D earth electrode with 3-D soil based on "shell" theory is introduced. Finally, based on the suggested method, the effect of abnormal resistance region (ARR) near DC earth electrode on electric field distribution is analyzed.
\end{abstract}

Keywords: DC earth electrode; electro-thermal coupling; abnormal resistance region (ARR); shell theory, finite element method

\section{Introduction}

When the high voltage direct current (HVDC) transmission system is working under mono-polar ground return mode, a high magnitude current will be injected into soil through the earth electrode. The injected current will cause the temperature rise of both earth electrode and soil, DC bias of transformer in nearby AC substations, DC corrosion on the underground metals pipeline and AC earth electrode nearby [1-4]. The DC electric field is influenced by the soil structure, injected current and earth electrode structure. So it is important to calculate the DC earth electrode electric field accurately and analyze the influence of soil structure and earth electrode on DC electrical field under-ground.

At present, the researches on the DC earth electrode mainly focus on three aspects: the DC earth electrode temperature rise process and the soil parameters' influences on the temperature rise process [5-8]; the transformer DC bias and underground metal DC corrosion based on circuit simulation software[9-10]; earth electrode electric field distribution law at complex soil structure[11-13]. In the researches about DC earth electrode electric field calculation, Ref [13] presents the modeling of DC earth electrode electric electrical field under complex tectonic, which takes DC earth pole as point source, thus reducing the amount of calculation, but this modeling is not suitable for the analysis of electrical field and thermal field near the earth electrode accurately. Refs [5-8] establish 3-D finite element DC earth pole modeling considering couple of electric field 
and thermal field. The result of this method is very accurate, at the same time huge unknown quantity will be generated; even there exists the problem of split singularity, when the large domain is considering. So it is essential to establish the modeling of DC grounding device both considering large domain and calculation accuracy. On the other hand, research about the effect of soil structure on DC earth electrode mainly focus on simple soil structure, such as level and vertical layered soil [11-12] or mountains and rivers [13], but there are very little researches about soils with massive texture.

The aim of this paper is to present a method for DC earth electrode electric field considering both grounding device structure and large disperse current domain. Due to the huge gap, sometimes up to 106, between large disperse current domain and very small section size of grounding conductor, the computation will be a sharp rise in traditional three-dimensional finite element method, and sometimes there will be singular point on interface of grounding conductor and soil in the process of FEM geometry subdivision. To solve the above problem, this paper introduces the "shell" theory [Error! Reference source not found.-16] into FEM method, which takes electrode conductor as 2-D surface in geometric model, as 3-D conductor with virtual thickness in FEM equation. Because of considering conduction of grounding conductor, the "shell" FEM method can calculate HVDC grounding device characteristic accurately, at the same time, the FEM computation in grounding conductor and soil adjacent domain have a decline attribute to the 2-D geometric model of grounding conductor. The high amplitude current which reaches several thousand amps flows through DC earth electrode chronically. Because of the conversion of electric energy into heat, the temperature of soil around earth electrode will raise, and then, the electrical and thermal parameters of soil changing correspondingly [5-8]. In order to simulate the actual working condition of DC earth electrode accurately, this paper set the electrical and thermal parameters of soil as function of temperature in the corresponding soil element and time. Finally, based on the wide applicability of FEM to any complex soil structure, this paper analyzes the influence of ARR near DC grounding on electric field distribution. Hope that conclusion of this paper can provide reference for actual DC grounding design.

\section{Numerical model of earth electrode system based on shell theory}

This section may be divided by subheadings. It should provide a concise and precise description of the experimental results, their interpretation as well as the experimental conclusions that can be drawn.

Current flowing process of DC earth electrode is coupling process between electric field and thermal field. They are satisfied with the constant electric field theory, Joule heating law and the basic principles of heat transfer. We calculate electric field and thermal field by FEM respectively, and then couple them.

\subsection{Finite element model of electric field}

Laplace's equation of constant electric field is used to describe DC earth pole electric field generated in the flowing process.

$$
\nabla^{2} \varphi=0
$$

$\varphi$ is the scalar potential. Formula (1) satisfies the following boundary conditions:

In the infinite distant place,

$$
\varphi=0 \quad r \rightarrow \infty
$$

On the earth surface,

$$
\gamma \cdot \vec{n} \cdot(\nabla \varphi)=0
$$

Where, $\vec{n}$ is the normal unit vector; $\gamma$ is the conductivity. 
The continuity conditions of potential and current density are satisfied at the earth grid and the soil continuum interface and the interface of different soil in complex soil.

In this paper, we use four-node tetrahedral unit to discrete solution domain, and use Galerkin weighted residual method and interpolation method to deal with the governing equations. Finally, the finite element equation of time $t$ is obtained,

$$
\boldsymbol{K}\{\varphi\}+\boldsymbol{P}=0
$$

The total stiffness matrix $K$ is related to the soil electrical conductivity; $\boldsymbol{P}$ is the quantity that is related to the injection current.

\subsection{Finite element model of thermal field}

According to the Joule heating law and heat transfer principle, the transient heat conduction equation of soil is as:

$$
k \cdot \nabla^{2} T+\boldsymbol{Q}-C_{v} \frac{\partial T}{\partial t}=0
$$

Where, $k$ represent the thermal conductivity of soil, $\mathrm{W} /(\mathrm{m} \cdot)$; $T$ is the temperature of each point in the soil, $; C_{\mathrm{v}}$ is volumetric heat capacity, $\mathrm{J} /\left(\mathrm{m}^{3}\right.$. ); $Q$ represents heat source density, and $\boldsymbol{Q}=\rho \boldsymbol{J}^{2}$, $\mathrm{W} / \mathrm{m}^{3}$, and it is determined by the electric current density parameter calculated by the electric field.

Formula (5) satisfies the following boundary conditions:

In the infinite distant place,

$$
T=T_{0}
$$

On the earth surface,

$$
k \frac{\partial T}{\partial n}=h_{0}\left(T_{0}-T\right)
$$

Where, $h_{0}$ and $T_{0}$ represent the ground heat transfer coefficient and ambient temperature.

Galerkin weighted residual method and interpolation method are used to deal with the governing equations. Finally, the finite element equation of time $t$ is obtained,

$$
\boldsymbol{N}\{T\}+\boldsymbol{C}\left\{\frac{\partial T}{\partial t}\right\}+\boldsymbol{F}=0
$$

The matrix $N, C, F$, respectively, is related to the thermal conductivity of $k$, specific heat capacity $C_{\text {v }}$ and heat source density $Q$.

\subsection{The introduction of thin shells theory}

Due to the huge gap, sometimes up to $10^{6}$, between large disperse current domain and very small section size of grounding conductor, the computation will be a sharp rise in traditional three-dimensional finite element method, and sometimes there will be singular point on interface of grounding conductor and soil in the process of FEM geometry subdivision. Therefore, this paper introduces thin shells theory to solve the above problem. The shell theory is originally used to study mechanical properties such as the deformation and internal force distribution of thin shell. As shown in Figure 1(a), it is ring pole with a virtual thickness d. And the triangular element is used to divide earth conductor surface, as shown in figure 1(b). 
4 of 13

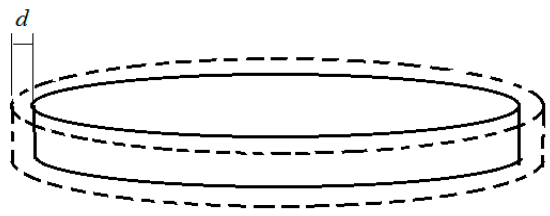

(a)

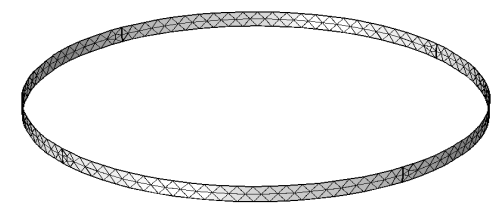

(b)

Figure 1. Schematic diagram of ground conductor model (a) 2-D earth conductor (b) Subdivision graph of 2-D earth conductor

we use four-node tetrahedral unit to discrete solution domain, and yield the following Galerkin residual equations of an element:

$$
\int_{S^{e}} N_{i}^{e}\left(d \gamma \nabla^{2} \varphi^{e}\right) \mathrm{d} S=0
$$

Where, $S$ e represents the surface integral unit interval; $d$ is the thickness of the shell equivalent unit; $\gamma$ is the conductivity of the earth conductor.

A scalar potential is expanded by nodal basis functions as:

$$
\varphi^{e}=\sum_{j=1}^{3} N_{j}^{e}(x, y) \varphi_{j}^{e}
$$

Substitution of (10) into (9) yields finite element equation of element e,

$$
\begin{aligned}
& R_{i}^{e}=\sum_{j=1}^{3} \varphi_{j}^{e} \int_{S^{e}}\left[d \gamma \nabla N_{i}^{e} \cdot \nabla N_{j}^{e}\right] \mathrm{d} S-I=0 \\
& (i=1,2,3)
\end{aligned}
$$

Combining formula (11) and formula (4), the finite element equations of the whole solution domain are obtained:

$$
\boldsymbol{K}^{*}\{\varphi\}+\boldsymbol{P}^{*}=0
$$

The same as the finite element equation of the current field is derived, equation (5) is treated by the Galerkin weighted residual method and the interpolation method,

$$
\begin{aligned}
& \sum_{j=1}^{3} d T_{j}^{e} \int_{S^{e}} k\left(\nabla N_{i}^{e} \cdot \nabla N_{j}^{e}\right) d S-\int_{S^{e}} d N_{i}^{e} \rho\left(J^{e}\right)^{2} d S \\
& +\sum_{j=1}^{3} \int_{S^{e}} d N_{i}^{e} C_{v} \frac{\partial T_{j}^{e}}{\partial t} N_{j}^{e} d S=0
\end{aligned}
$$

Combining formula (14) and formula (8), the finite element equations of the whole solution domain are obtained:

$$
\boldsymbol{N}^{*}\{T\}+\boldsymbol{C}^{*}\left\{\frac{\partial T}{\partial t}\right\}+\boldsymbol{F}^{*}=0
$$

From the above derivation, in the drawing of geometric model and process of FEM geometry subdivision, the electrode conductor is equivalent to 2-D surface, which can greatly simplify the mesh generation process and reduce computation, specially in large solution area. In FEM equation(11) and (13), the virtual thickness $d$ is cleverly introduced, so the calculated results are equivalent to the volume fraction of the three prism element with a triangle bottom and a thickness $\mathrm{d}$. The above method can not only reflect diffuser function of earth conductor to ensure calculation accuracy, but also solve the problem of huge computation in large solution area. In addition, due to the contradiction between limited computational resources of finite element method and the infinite flowing space, this paper introduces the space coordinate transformation in the infinite soil region far away from the grounding grid to simplify calculation [17]. 


\subsection{Coupling process of electric and thermal fields}

According to [6], the electrical and thermal parameters of soil show obvious dynamic temperature characteristics with temperature, which are expressed by function $\rho(T), C(T)$ and $k(T)$. The algorithm can directly reflect the actual working mechanism of DC earth electrode by coupling of electric field and thermal field, and provide theoretical basis for practical ground design. Because the finite element equation of temperature field contains the temperature to the time partial differential, we uses the time domain difference method [5] to deal with it. The following is the coupling process of electric field and thermal field:

(1) when $t=t_{1}$, firstly, soil conductivity $\rho_{1}=\rho_{0}$ (initial value), the electric field calculation matrix of time t1:

$$
\boldsymbol{K}_{0}^{*}\{\varphi\}+\boldsymbol{P}^{*}=0
$$

$K_{0}{ }^{*}$ indicates that the resistivity in this matrix is set to the resistivity in initial time.

Secondly, the heat source density $Q_{1}$ at $t_{1}$ time is determined by current density $J_{1}$ calculated by the last step, $\boldsymbol{Q}_{1}=\rho \boldsymbol{J}_{1}^{2}$.The specific heat capacity is $C_{0}$ (initial value); the thermal conductivity is $k_{0}$ (initial value); And the method of time domain difference is used to deal with the transient temperature field. calculation matrix of thermal field:

$$
\begin{aligned}
& \{T\}_{1}=\frac{\Delta T}{3}\left(\boldsymbol{I}+\frac{2 \Delta T}{3} \boldsymbol{C}_{0}^{*-1} \boldsymbol{N}_{0}^{*}\right)^{-1}\left(2 \boldsymbol{F}^{*}{ }_{1}+\boldsymbol{F}^{*}{ }_{0}\right) \\
& +\left(\boldsymbol{I}+\frac{2 \Delta T}{3} \boldsymbol{C}_{0}^{*-1} \boldsymbol{N}_{0}^{*}\right)^{-1}\left(\boldsymbol{I}-\frac{\Delta T}{3} \boldsymbol{C}_{0}^{*-1} \boldsymbol{N}_{0}^{*}\right)\{T\}_{0}
\end{aligned}
$$

$F_{1}{ }^{*}$ indicates that the heat source density in this matrix is set to function of current density in $t_{1}$ time.

(2) when $t=t_{2}$, the electrical conductivity were determined by temperature $T$ in $t_{2}$ time,

$$
\boldsymbol{K}_{1}^{*}\{\varphi\}+\boldsymbol{P}^{*}=0
$$

The heat source density $Q_{2}$ at t 2 time is determined by current density $J_{2}$ calculated by the last step, $\boldsymbol{Q}_{2}=\rho \boldsymbol{J}_{2}^{2}$.The specific heat capacity is $C_{1}$; the thermal conductivity is $k_{1}$,

$$
\begin{aligned}
& \{T\}_{2}=\frac{\Delta T}{3}\left(\boldsymbol{I}+\frac{2 \Delta T}{3} \boldsymbol{C}_{1}^{*-1} \boldsymbol{K}_{1}^{*}\right)^{-1}\left(2 \boldsymbol{F}^{*}{ }_{2}+\boldsymbol{F}_{1}^{*}\right) \\
& +\left(\boldsymbol{I}+\frac{2 \Delta T}{3} \boldsymbol{C}_{1}^{*-1} \boldsymbol{K}_{1}^{*}\right)^{-1}\left(\boldsymbol{I}-\frac{\Delta T}{3} \boldsymbol{C}_{1}^{*-1} \boldsymbol{K}_{1}^{*}\right)\{T\}_{1}
\end{aligned}
$$

Summing up the above calculation process, the calculation matrix of electric field and thermal field is obtained by analogy,

$$
\begin{gathered}
\boldsymbol{K}_{n-1}^{*}\{\varphi\}+\boldsymbol{P}^{*}=0 \\
\{T\}_{n}=\frac{\Delta T}{3}\left(\boldsymbol{I}+\frac{2 \Delta T}{3} \boldsymbol{C}_{n-1}^{*-1} \boldsymbol{N}_{n-1}^{*}\right)^{-1}\left(2 \boldsymbol{F}^{*}{ }_{n}+\boldsymbol{F}^{*}{ }_{n-1}\right) \\
+\left(\boldsymbol{I}+\frac{2 \Delta T}{3} \boldsymbol{C}_{n-1}^{*-1} \boldsymbol{N}_{n-1}^{*}\right)^{-1}\left(\boldsymbol{I}-\frac{\Delta T}{3} \boldsymbol{C}_{n-1}^{*}{ }^{-1} \boldsymbol{N}_{n-1}^{*}\right)\{T\}_{n-1}
\end{gathered}
$$

This method can accurately simulate the time variation of soil electrical and thermal parameters, and realize the dynamic coupling process between electric field and thermal field.

\section{Verification}

According to [18], DC earth electrode, a single ring with radius of $255 \mathrm{~m}$, was placed into the layered soil, and the buried depth is $2.5 \mathrm{~m}$. Besides the parameters of soil are shown in Table 1 . The resistivity, volumetric heat capacity and density of the coke, soil and pole are shown in Table 2. According to [5], the fitting formula of $\rho(T), C(T)$ and $k(T)$ changing with temperature, are shown as formula (21), (22) and (23). 
6 of 13

$$
\begin{gathered}
\rho(T)=\left\{\begin{array}{c}
16.5, \mathrm{~T} \in\left(20^{\circ} \mathrm{C}, 44^{\circ} \mathrm{C}\right) \\
0.0055 \times 2.718^{\wedge}(0.182 \times \mathrm{T}), \mathrm{T} \in\left(44^{\circ} \mathrm{C}, 90^{\circ} \mathrm{C}\right)
\end{array}\right. \\
c(T)=\left\{\begin{array}{l}
-0.008617 \times T^{3}+0.6859 \times T^{2}-18.83 \times T^{1}+1130, T \in\left(20^{\circ} \mathrm{C}, 55^{\circ} \mathrm{C}\right) \\
-0.001478 \times T^{3}+0.4289 \times T^{2}-46.22 \times T^{1}+2216, T \in\left(55^{\circ} \mathrm{C}, 90^{\circ} \mathrm{C}\right)
\end{array}\right. \\
\mathrm{k}(T)=\left\{\begin{array}{c}
-2.817 \times 10^{-6} \times T^{3}+0.000155 \times T^{2}-0.002711 \times T^{1}+1.243, T \in\left(20^{\circ} \mathrm{C}, 55^{\circ} \mathrm{C}\right) \\
3.187 \times 10^{-5} \times T^{3}-0.006339 \times T^{2}+0.3805 \times T^{1}-5.93, T \in\left(55^{\circ} \mathrm{C}, 90^{\circ} \mathrm{C}\right)
\end{array}\right.
\end{gathered}
$$

We set the 1200A current for DC earth electrode. Table 3 lists the calculated results which include calculation results obtained by the method of this paper, by International recognized Grounding Calculation Software (CDEGS) and by actual measurement in [7]. The comparison result shows that the algorithm is effective.

Table 1. The soil around the grounding level stratification parameter

\begin{tabular}{ccc}
\hline Soil layers & Resistivity $(\mathbf{\Omega} \cdot \mathbf{m})$ & Thickness $(\mathbf{m})$ \\
\hline 1 & $\rho(T)$ & 9.7 \\
2 & 48.2 & 27.1 \\
3 & 13.2 & Infinite \\
\hline
\end{tabular}

Table 2. Parameters of calculated model

\begin{tabular}{cccc}
\hline parameters & soil & coke & pole \\
\hline density $/\left(\mathrm{Kg} \cdot \mathrm{m}^{3}\right)$ & 2700 & 1100 & 4750 \\
Resistivity $/(\Omega \cdot \mathrm{m})$ & $\mathrm{Tab} .1$ & 0.3 & $1.7 \times 10-7$ \\
volumetric heat capacity & $c(T)$ & 800 & 450 \\
$/\left(\mathrm{J} \cdot(\mathrm{Kg} \cdot \mathrm{K})^{-1}\right)$ & & & 44.5 \\
thermal conductivity & $k(T)$ & 4 & \\
$/\left(\mathrm{W} \cdot(\mathrm{m} \cdot \mathrm{K})^{-1}\right)$ & & & \\
\hline
\end{tabular}

Table 3. Results of comparing mode

\begin{tabular}{ccc}
\hline Comparison & Resistance/ $\boldsymbol{\Omega}$ & $\begin{array}{c}\text { Maximum temperature } \\
\text { rise after 20d / }\end{array}$ \\
\hline MEA & 0.025 & Almost unchanged \\
CDEGS & 0.021 & 0.46 \\
FEM & 0.022 & \\
\hline
\end{tabular}

\section{The influence analysis of arr on the dc earth electrode system}

In order to analyze the influences of ARR's position on DC earth pole, we establish a single ring earth electrode model under an uniform soil condition. The model's ring diameter is $1000 \mathrm{~m}$, and the initial soil resistivity is $80 \Omega \cdot \mathrm{m}$. Using a $300 \mathrm{~m} \times 600 \mathrm{~m} \times 70 \mathrm{~m}$ rectangular block to simulate the ARR, the resistivity of the low resistivity-ARR and the high resistivity-ARR are setted $5 \Omega \cdot \mathrm{m}$ and $500 \Omega \cdot \mathrm{m}$, respectively. Finally, we analyze the influence of high and low resistivity-ARR at different horizontal positions and different depth on electric field distribution. 


\subsection{The influences of $A R R$ 's horizontal position on earth electrode system}

In the vertical direction, The upper surface of ARR is flat with the earth surface. In the horizontal direction, ARR in the center of earth electrode and $150 \mathrm{~m}, 300 \mathrm{~m}, 450 \mathrm{~m}, 600 \mathrm{~m}, 750 \mathrm{~m}, 900 \mathrm{~m}$ away from it in $\mathrm{x}$ axis are calculated and modeled separately, numbered from model 2 to model 8. And model 1 is the case of no ARR(namely uniform soil).

Surface potential distribution 2-D graph and surface potential distribution curve( a line which goes through the center of the circle earth electrode and is perpendicular to the long edge of the ARR ) of model 1-8 under soil structure with low resistivity-ARR $(5 \Omega \cdot m)$ are separately listed in Figure 2(a) and (b). Figure 3 shows the current density distribution of model 1-8. Conclusions from figure 2 (a) are that, compared with that under uniform soil conditions, the surface potential under soil structure with low resistivity-ARR significantly reduces; If the position of low resistivity-ARR is different, degree of surface potential amplitude reduction also is different; When a part of the earth conductor is in low resistivity-ARR, reduction of surface potential is most obvious; And the more a part of the earth conductor that is located in low resistivity-ARR is, the more surface potential drops. For example, compared with that under uniform soil condition, the surface potential of model 5 was decreased by $22.75 \%$.

As shown in Figure 2 (b), the following laws can be obtained: Compared with the uniform soil, the changing trend of the potential on the low resistivity-ARR is more moderate; When the low resistivity-ARR is located in the ring of ring earth pole(namely model 2 to 3), the surface potential distribution curve is basically consistent with that of model 1 and only fells by less than $10 \%$, besides, the potential on low resistivity-ARR raises. Because the low soil resistivity region has the ability to attract current, and the lower the resistivity is, the stronger the ability to attract current is[1]; And from Figure 3, because the low resistivity area has the ability to attract current, the current density of ARR ( model 2 to 3) is increased, so the potential on ARR is increased. Compared with model 1, When a part of the earth conductor is in low resistivity-ARR(namely model 4 to 6 ), surface potential amplitude of model 4 to 6 is significantly decreased and fells by about $20 \%$, besides, the potential on low resistivity-ARR raises; The reason is that, the more ARR is close to the earth conductor, the more current it can attract is, as shown in figure 3. Therefore, the more current flows through the region with lower resistivity, which leads to the overall potential decreasing and the potential on ARR rising. When ARR is outside the ring and far away from the earth pole(model 7 to 8 ), the surface potential distribution curve is basically consistent with that of model 1 and only fells by about $10 \%$, and the changing trend of the potential on ARR is more moderate; We combine Figure 3 to analyze the reason. From it, the phenomenon of the low resistivity-ARR attracting current (model 7-8) is still obvious; However, compared with that of model 4 to 6 , the current it attracts is less, so low resistivity-ARR of model 7 to 8 has little effect on the flowing capacity of the DC earth electrode. Which leads to the change of the surface potential distribution of model 7 to 8 is not obvious, and is almost coincident with that under uniform soil condition
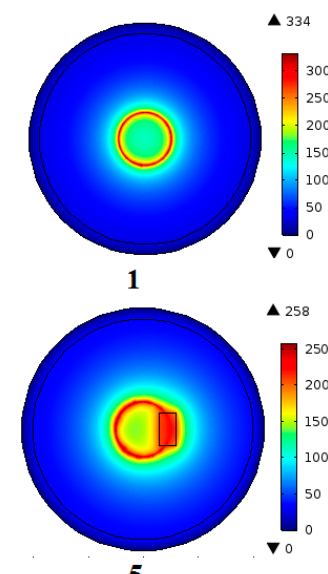

$\mathbf{5}$

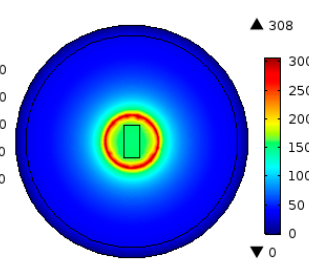

2

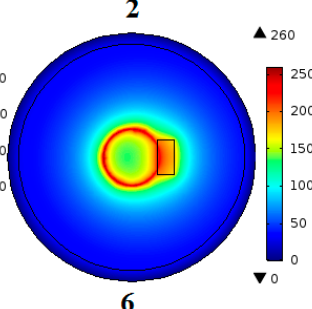

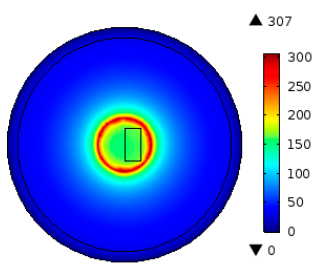

3

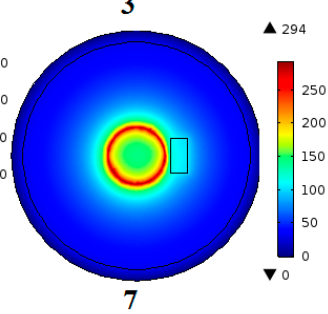

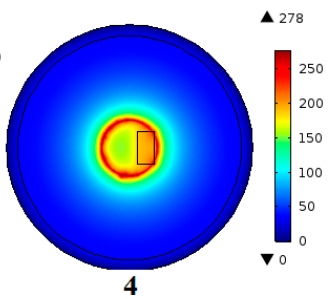

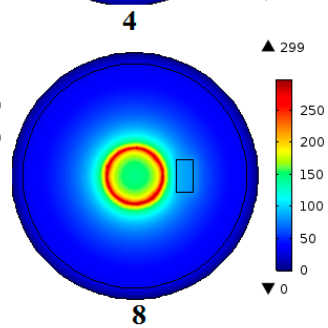

(a) 


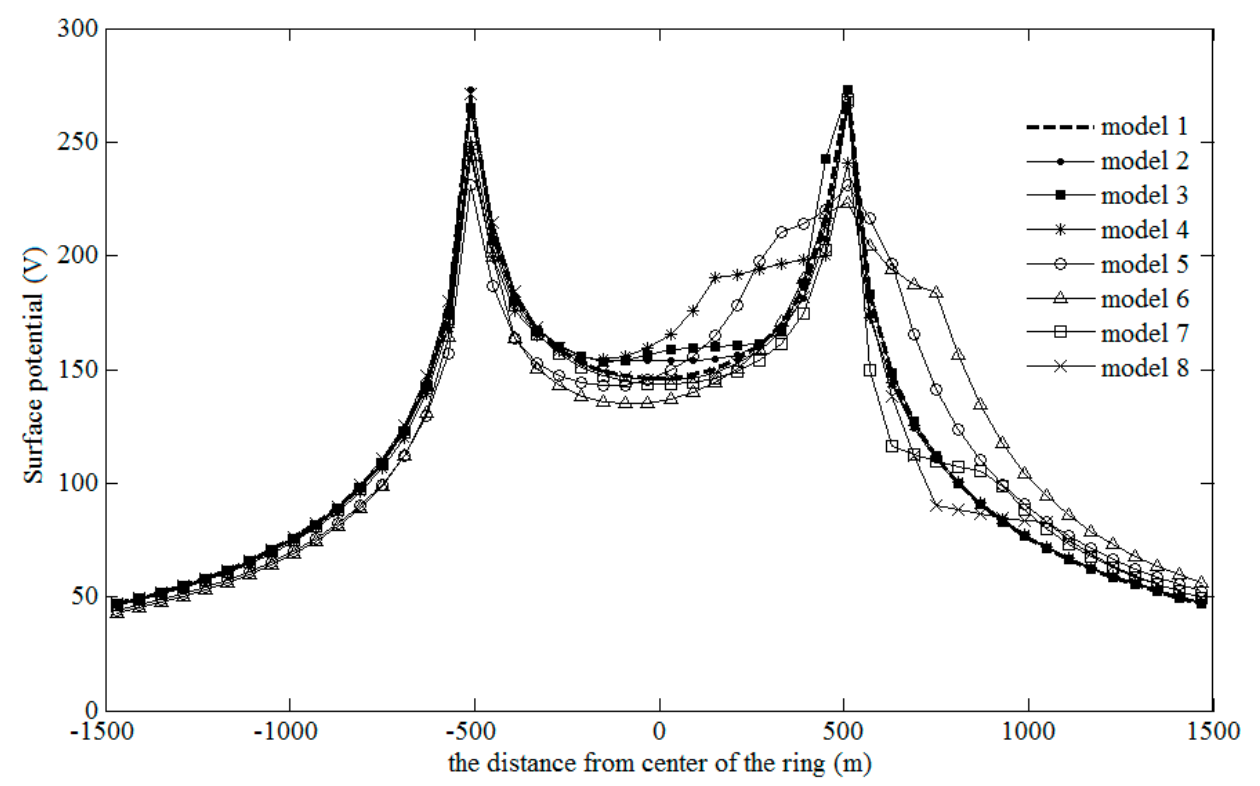

(b)

Figure 2. surface potential distribution at different horizontal position of low resistivity-ARR ( a) Surface potential distribution 2-D graph( b) surface potential

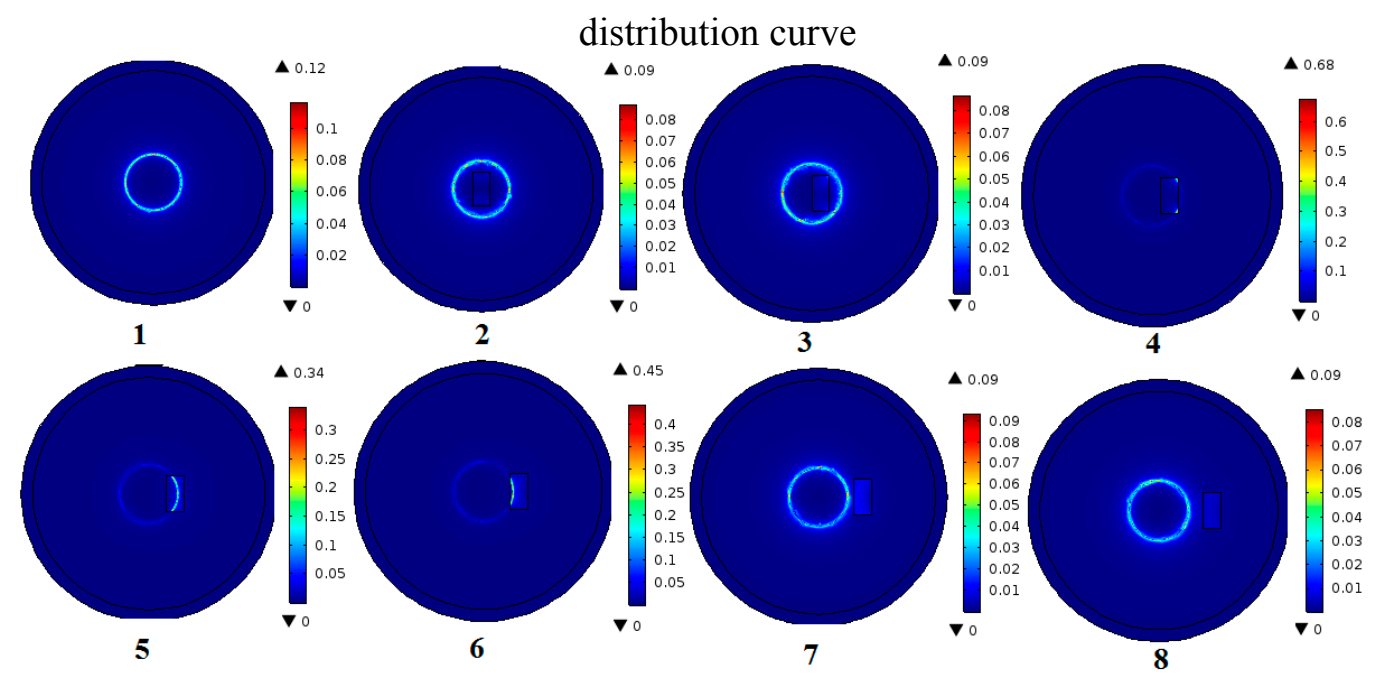

Figure 3. current density distribution at different horizontal position of low resistivity-ARR

Surface potential distribution 2-D graph and surface potential distribution curve( a line which goes through the center of the circle earth electrode and is perpendicular to the long edge of the ARR) of model 1-8 under soil structure with high resistivity-ARR $(500 \Omega \cdot \mathrm{m})$ are separately listed in Figure 4(a) and (b). Figure 5 shows the current density distribution of model 1 to 8 . Conclusions from figure 4 (a) are that, compared with that under uniform soil condition, the surface potential under soil structure with high resistivity-ARR significantly reduces; If the position of high resistivity-ARR is different, degree of surface potential amplitude rise also is different; When a part of the grounding conductor is in high resistivity-ARR, increasing of surface potential is most obvious; And the more a part of the earth conductor that is located in ARR is ,the more surface potential rises. For example, compared with that under uniform soil condition, the surface potential of model 5 was increased by $19.46 \%$.

As shown in Figure 4 (b), it is the surface potential distribution curve of model 1 to 8 . The following laws can be obtained: Compared with uniform soil, the changing trend of potential on 
the high resistivity-ARR is more steep; When the high resistivity-ARR is located inside the ring of ring grounding pole(namely model 2 to 3 ), the surface potential distribution curve is basically consistent with that of model 1 and only rises by less than $10 \%$, besides, the potential on high resistivity-ARR drops. Because the high soil resistivity region has the ability to reject current, and the higher the resistivity is, the stronger the ability to reject current is[1]. So the current density of ARR ( model 2 to 3 ) is decreased, and the potential on ARR goes down. Compared with model 1, when a part of the earth conductor is in high resistivity-ARR(namely model 4 to 6), surface potential amplitude of model 4 to 6 is significantly increased and rises by about $20 \%$, besides, the potential above high resistivity-ARR fells. We combine Figure 4 to analyze the reason. From it, the rejection of high resistivity-ARR to the current is obvious, which leads to diffuse capacity of earth conductor located inside the ARR weakening. It can be equivalent to the effective flowing length of the earth conductor becomes shorter, so the whole surface potential is significantly increased; Besides, the rejection of high resistivity-ARR to the current leads to the decreasing of the current density, so the surface potential on high resistivity-ARR drops. When ARR is outside the ring and far away from the earth pole(model 7 to 8 ), the surface potential distribution curve is basically consistent with that of model 1 and only fells by about $6 \%$, and the changing trend of the potential on ARR is more moderate; The reason is that, high resistivity-ARR is farther from the earth conductor, so the rejection of the current is weakened. Which leads to the effect of high resistivity-ARR on the whole electric potential distribution is small.

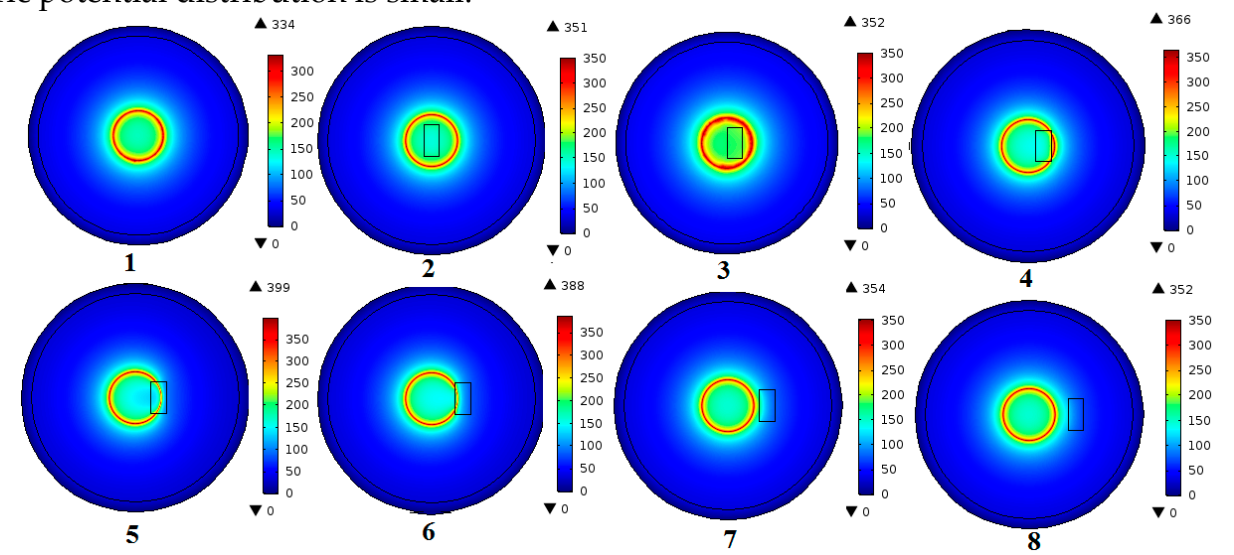

(a)

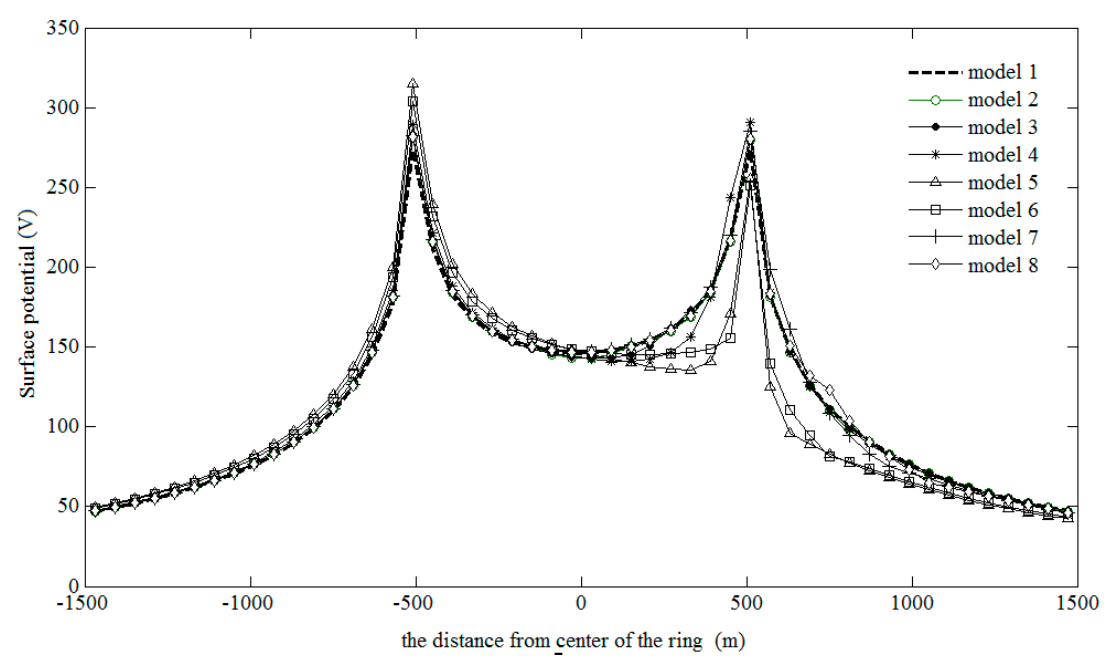

(b)

Figure 4. surface potential distribution at different horizontal position of high resistivity-ARR ( a) Surface potential distribution 2-D graph ( b) surface potential distribution curve 


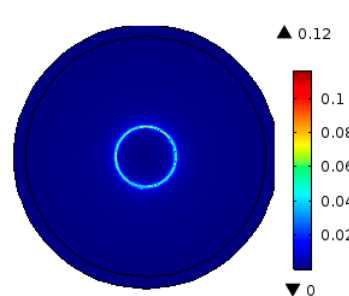

1
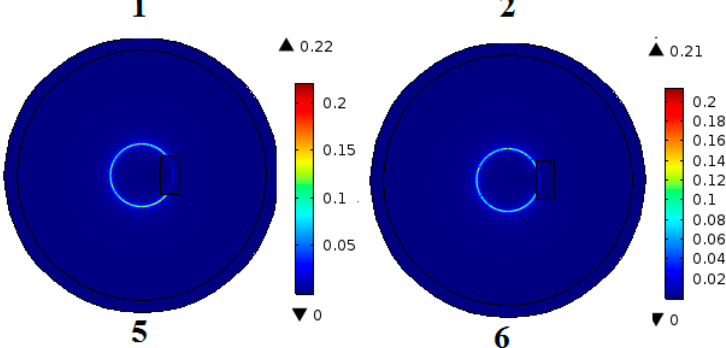

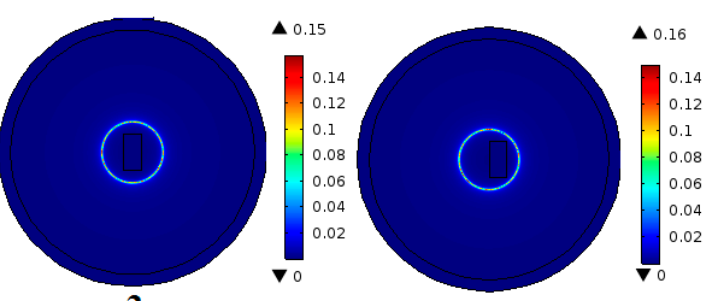

3

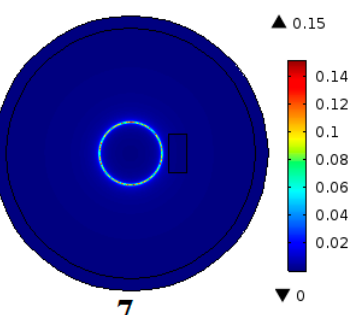

7

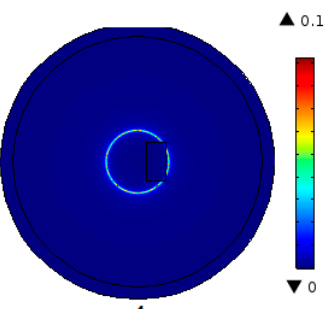

4

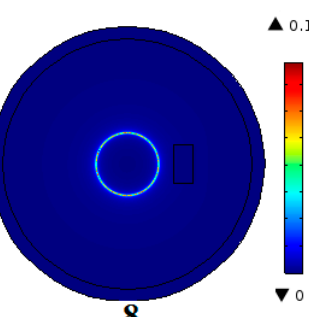

Figure 5. current density distribution at different horizontal position of high resistivity-ARR

Earthing resistance is an important index in DC grounding design, so it is necessary for us to analyze the earthing resistance at different horizontal position of ARR.

Figure 6 shows the earthing resistance of low and high resistivity-ARR at different horizontal position. As can be seen from the figure, when the position of ARR is different, earthing resistance is also different. Showing earthing resistance is decreased under low resistivity-ARR, while that is increased under high resistivity-ARR; When a part of the earth conductor is in ARR, the changing of earthing resistance is biggest. Compared with that under uniform soil, the earthing resistance under the soil condition with low resistivity-ARR is decreased by $24.56 \%$, while it is increased by $31.58 \%$ under the soil condition with high resistivity-ARR. Thus, the influence of ARR on earthing resistance can not be ignored. Especially, step potential and earthing resistance are both increased in high resistivity-ARR. Therefore, the influence of the low or high resistivity-ARR on the earth electrode system should be evaluated in the actual earth electrode design.

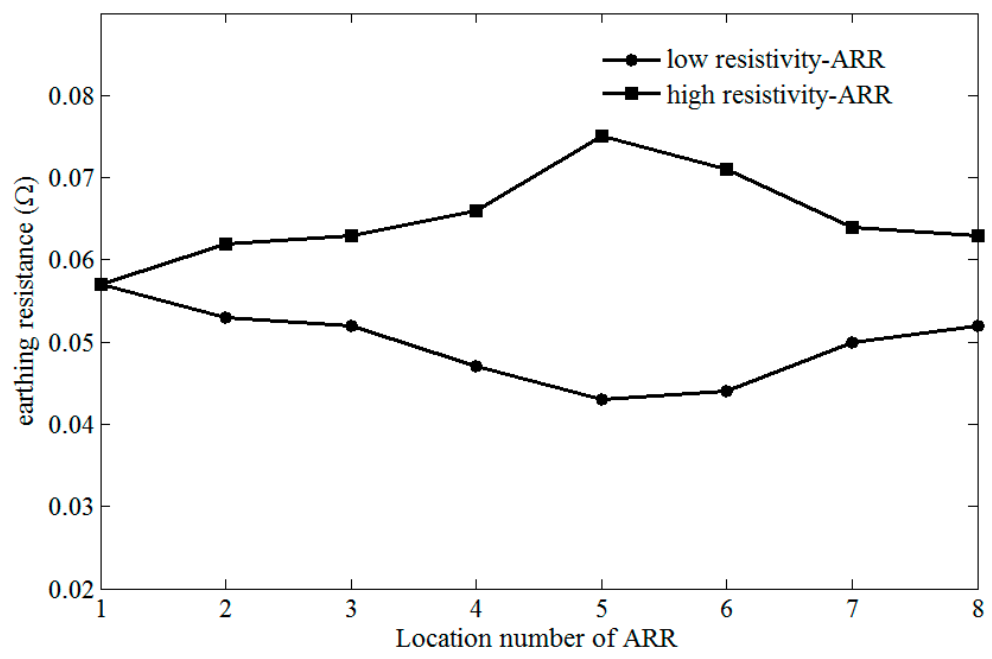

Figure 6. earthing resistance of different surface abnormal resistance position

\subsection{The influence of $A R R$ 's vertical depth on earth electrode system}

Due to the large range, direct current flowing in soil, the influence of deep soil structure on the DC earth electrode can not be ignored. So it is necessary to analyze the influence of ARR's vertical depth on electric field distribution. In the case of ARR located at a distance from the ground surface to $0 \mathrm{~m}, 20 \mathrm{~m}, 40 \mathrm{~m}, 60 \mathrm{~m}, 80 \mathrm{~m}, 100 \mathrm{~m}$ and $150 \mathrm{~m}$, the calculation results, that is surface potential distribution of low resistivity-ARR and high resistivity-ARR, show in Figure 7 and figure 8. From 
figure 7, the following laws can be obtained: When low resistivity-ARR is located in different vertical depth, influence degree of it on the surface potential is different; Especially, when the low resistivity-ARR is located in the surface, the influence on the surface potential distribution is greatest. When it is located at a distance from the ground surface to $20 \mathrm{~m}, 40 \mathrm{~m}, 60 \mathrm{~m}, 80 \mathrm{~m}, 100 \mathrm{~m}$ and $150 \mathrm{~m}$, surface potential distribution is slightly lower than that under uniform soil. The reason is that the ability of low resistivity region to attract current makes the current more easily flowing into low resistivity-ARR in the depths of soil, so surface current density and the surface potential is decreased. From figure 8, the influence of high resistivity-ARR at different vertical depths on surface potential is different; Particularly, when ARR is located on the surface of the earth, the influence is greatest; When it is located below the ground $20 \mathrm{~m}, 40 \mathrm{~m}, 60 \mathrm{~m}, 80 \mathrm{~m}, 100 \mathrm{~m}$ and $150 \mathrm{~m}$, the surface potential distribution is slightly higher than that under uniform soil. And phenomenon of potential rise on ARR is more obvious. The reason is that the ability of low resistivity region rejecting current makes the current mainly gathering in the area on ARR, so current density on ARR increases, and then the potential rises.

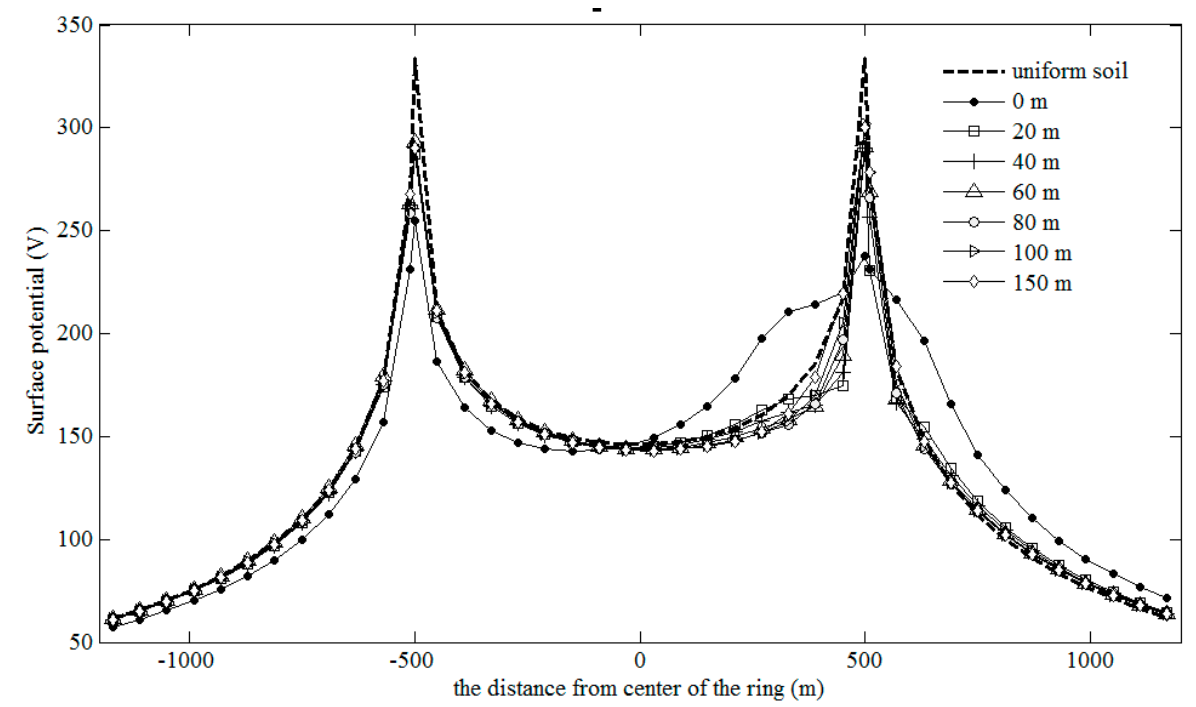

Figure 7. Surface potential distribution of low resistivity-ARR at different depths

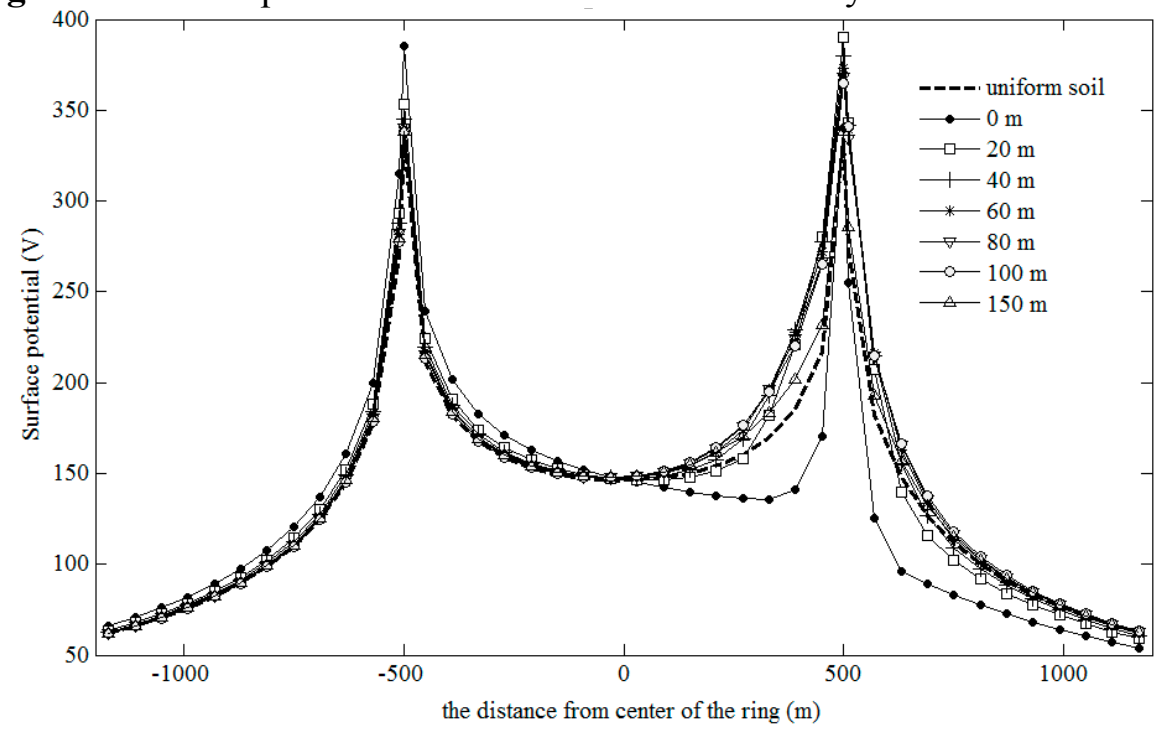

Figure 8. Surface potential distribution of high resistivity-ARR at different depths

Figure 9 shows the earthing resistance at different ARR's vertical depths. That can be obtained: Compared with the condition of high resistivity-ARR, earthing resistance under soil with low resistivity-ARR is low. When the vertical depth of ARR is $150 \mathrm{~m}$, the gap between them still is $19.24 \%$; With the increase of the ARR's depth, the earthing resistance of them will eventually tend to be 
stable; Compared with the $0.057 \Omega$ of the uniform soil, the earthing resistance of low resistivity-ARR at the $150 \mathrm{~m}$ vertical depth, is reduced by $11.03 \%$, and the high resistivity-ARR is increased by $10.16 \%$. It can be known that, when the vertical depth of ARR is deeper, the influence on ground resistance can reach about $10 \%$; It is necessary to survey the deep soil geological condition at practical earth electrode design.

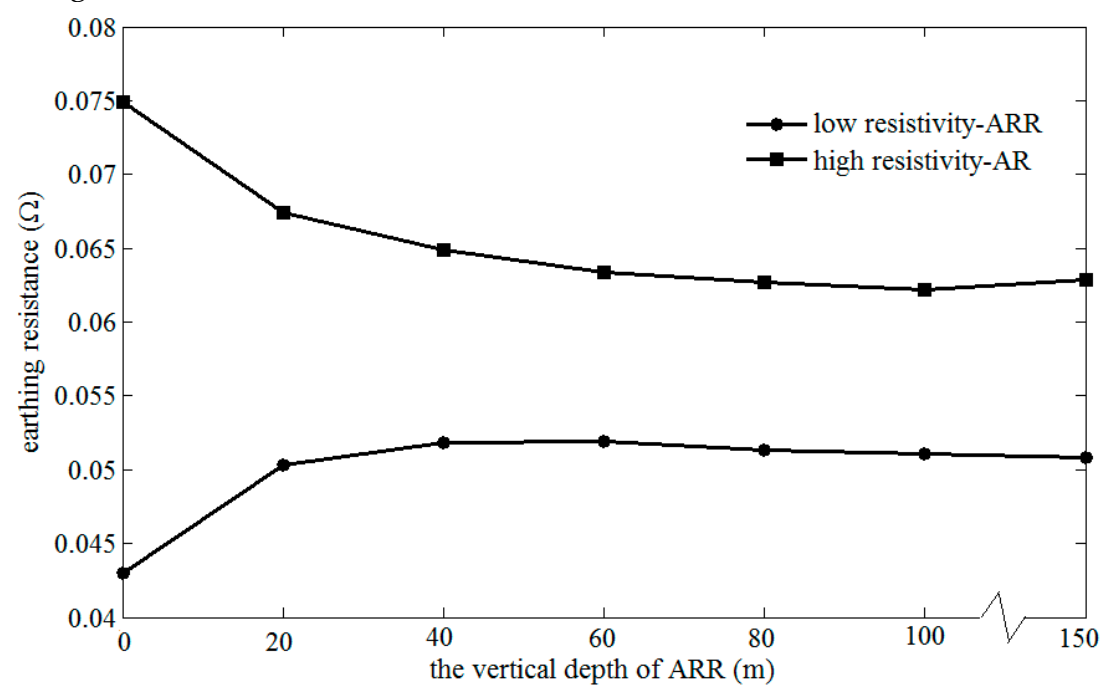

Figure 9. earthing resistance of ARR at different depths

\section{Conclusions}

This paper establishes finite element model of the DC earth electrode Considering massive ARR based on shell theory. And analyzes the influence of ARR at different horizontal positions and different depth on electric field distribution. The following rules are obtained:

(1) The more ARR is close to the earth conductor, the bigger influence of ARR on Surface potential is; Besides, when ARR is close to the earth conductor, there is a certain influence on the whole surface electric potential distribution; However, when ARR is far away from the earth electrode, only part surface potential distribution is affected.

(2) The more low resistivity-ARR is close to the ground conductor, the more obvious earthing resistance is decreased; On the contrary, the more high resistivity-ARR is close to the ground conductor, the more obvious ground resistance is increased.

In summary, the influence of ARR at different horizontal positions and different depth on DC earth electrode parameters can not be ignored. In the actual design, a detailed survey on geological conditions of the polar site near the DC earth electrode should be done. Considering massive ARR, analyzes the influence of ARR near DC earth electrode on electric field distribution. Hope that conclusion of this paper can provide reference for actual DC earth electrode design.

Acknowledgments: The authors wish to thank National Natural Science Foundation of China (Grant No. 51307152) for their financial support.

Author Contributions: Jingli Li, Liying Guo conceived and designed the simulations; Liying Guo and Yuanbo Li performed the simulations; all authors analyzed the data; Jingli Li, Liying Guo wrote the paper, while other authors offered their modification suggestions for the manuscript.

Conflicts of Interest: The authors declare no conflict of interest.

\section{References}

1. He, J.L.; Zeng, R. Grounding technology of electric power system. Science Press: Beijing, Chına, 2007.

2. Liang, X.M.; Zhang, P; Chang, Y. Recent Advances in High-Voltage Direct-Current Power Transmission and Its Developing. Potential pwr. sys. Tech. 2012, 36, 1-9. 
3. Rao H.; L1 Y. Study on Four HVDC Systems Sharing a Common Ground Electrode. High Vol. Eng. 2015, 41, 3672-3678.

4. Zhao J.; He J.L. Analysis on the common grounding electrodemode for UHVDC and HVDC power transmission systems. Elec. Pwr. 2007, 40, 45-47.

5. Yuan Tao.; Luo L.; Yang Q.; Sima W.X.; Wang J.D. Analysis on the Temperature Characteristic of Soil and Its Influence onthe Cirque Grounding Electrode Heating. Proc. CSEE. 2013, 33, 188-195.

6. Zhang T.; Tan X.R.; Huang Y.H. Analysis on Transient Temperature Rise of UHVDC Common Grounding Electrode. High Vol. Eng. 2015, 41, 3672-3678.

7. Du H.Z.,Wen X.S. Test and Simulation for Horizontal Ground Electrode's Temperature Rise. High Vol. Eng. 2013, 39, 1184-1190.

8. Wang Y.; Li X.P. Test and Computation for Vertical Ground Electrode's Transient Temperature Rise. Proc. CSEE. 2013, 33, 184-190.

9. Wang X.X.; Ruan L.;Wen X.S.; Quan Y.T. Characteristics Analysis of DC-bias Currents Considering Deep-earth Resistivity. High Vol. Eng. 2015, 41, 1536-1543.

10. Chi X.H.; Zhang Y.J. Protective Distance between HVDC Electrode and Underground Metal Pipeline. Pwr. Sys. Tech. 2008, 32, 111-115.

11. Ruan L.; Quan J.T.; Yang X.K. Influence of Deep Earth Resistivity on Direct Current Distribution in AC Power Grid. High Vol. Eng. 2014, 40, 3528-3536.

12. Fu Z.X.; Tan H.D.; Liu H.F. Potential Numerical Simulation and Analysis of Influencing Factors ofToroidal HVDC Grounding Electrode. Pwr. Sys. Tech. 2016, 40, 1909-1915.

13. Ma J.J. Ground Potential Distribution of HVDC Ground Electrode Considering Tectonics. Master's thesis, North China Electric Power University, Beijing, China, 2012.

14. Lei L. Shell vector elements based numerical methods for electromagnetic scttering. PH.D. thesis, University of Electronic Science and technology, Chengdu, China, 2012.

15. E. Abenius, F. Edelvik. Thin Sheet Modeling Using Shell Elements in the Finite-Element Time-Domain Method. IEEE Trans. Antenn. Propag., 2006, 54, 28-34.

16. Lei L.; Hu J.; Hu H.Q. Solving Scattering from Conducting Body Coated by Thin-Layer Material by Hybrid Shell Vector Element with Boundary Integral Method. Intl. J Antenn. Propag. 2012, pp: 1-9.

17. Li J.L.; Jiang J.D.; Li L.L. Simulation and Experiment Study on Resistance-Reducing Mechanism of Grounding Device With Spicules. Pwr. sys. Tech. 2013, 37, 212-217.

18. Zhang L.; Su L. Parametric Computation and Measurement of Jiangcheng HVDC Grounding Electrode. Hubei electric power, 2015, 39, 39-41.

(c) 2017 by the authors. Licensee Preprints, Basel, Switzerland. This article is an open access article distributed under the terms and conditions of the Creative Commons by Attribution (CC-BY) license (http://creativecommons.org/licenses/by/4.0/). 